\title{
Perfilado psicosocial para la intervención socioeducativa en los servicios sociales
}

\section{Psychosocial profiling for social service users}

\author{
Lucía Martínez Virto ${ }^{1}$
}

\begin{abstract}
${ }^{1}$ Profesora Titular de Trabajo Social y Servicios Sociales del Departamento de Sociología y Trabajo Social, Universidad Pública de Navarra (España). E-mail: lucia.martinez@unavarra.es
\end{abstract}

\begin{abstract}
El trabajo que aquí se presenta ha sido realizado el marco de la evaluación del Programa de Incorporación de los Servicios Sociales de la ciudad de Pamplona, capital de la Comunidad Foral de Navarra, situada al norte de España. El trabajo contó con el apoyo y financiación del Ayuntamiento de Pamplona.

La autora agradece a las profesionales del Programa de Incorporación Social del Ayuntamiento de Pamplona la reflexión y sistematización realizada.
\end{abstract}

Ayuntamiento de Pamplona.

Universidad Pública de Navarra.

Pamplona (Navarra), España.

\section{Resumen}

Este artículo sistematiza la experiencia de perfilado de personas usuarias de los servicios sociales de Pamplona, ciudad al norte de España. Tras un año de trabajo, el equipo multidisciplinar del programa ha logrado perfilar la población atendida e identificar los criterios de atención y la frecuencia del seguimiento. El resultado ha sido la clasificación de tres programas de atención: (1) programa de incorporación con atención básica, (2) programa de acompañamiento socioeducativo y (3) unidad de apoyo psicológico. En cada uno de estos programas se identifican hasta siete tipos distintos de perfiles psicosociales. A partir de esta estructura y criterios, se han definido tres grados de intensidad en la atención: alta, media y baja, que requieren metodologías de acompañamiento, seguimiento y frecuencia distintas. Esta experiencia aporta dos resultados con grandes potencialidades para el sector profesional y académico de ámbito psicosocial y socioeducativo. Por un lado, evalúa de manera multidisciplinar el modelo de intervención socioeducativa con las personas en exclusión social. Por otro, sistematiza y comparte un modelo consensuado para la intervención desde los servicios sociales. La literatura internacional y estatal en los campos de la inclusión social comparte retos y desafíos. Por ello, esta sistematización aporta criterios técnicos, bases teóricas, enfoques y metodología que seguro arrojan luz a dilemas profesionales presentes en otros territorios.

Palabras clave: servicios sociales, exclusión social, perfilado, socioeducativo, sistematización.

\begin{abstract}
This article systematizes the profiling experience of social service users in Pamplona, a city in northern Spain. The economic crises in Spain had a strong psychosocial impact on people affected by unemployment or job insecurity. After a decade of the serious crisis
\end{abstract}


that started in 2008, at least one out of ten homes in Spain has experienced a mental health disorder or depression. For this reason, an important volume of social service users in Spain accumulate situations of poverty and psychosocial deterioration, especially in the field of social incorporation.

This involves a strong pressure on the services with an increasing volume of people with a diversity of needs and psychosocial profiles, ultimately impacting in the fight against poverty and social exclusion. Faced with this situation, the professionals of the Social Services of Pamplona began a multidisciplinary work process to define and systematize the intensity and focus on social service users. 19 professionals (the total of professionals in the area) from different disciplines related to psychosocial and socio-educational care (social educators, social workers, psychologists) participated in this process. After a year of collaborative work, they managed to outline the population and identify support criteria and follow-up frequency. The results introduce the classification of three care programs: (1) incorporation program with basic care, (2) socio-educational support program and (3) psychological support unit. Up to 7 different types of psychosocial profiles were identified in each of these programs. Based on this structure and the criteria, three degrees of care were defined: high, medium and low intensity, which require different support, monitoring and frequency methodologies. This intensity takes into account the level of psychosocial deterioration social service users.

To establish these criteria, social work professionals have utilized a variety of diagnostic tools in the intervention areas of employment, health and consumption, care or personal and family situation. They also supported the analysis with a diagnosis of social skills in education and training.

Social care professions, such as Psychology or Social Work, were born from the alliance between theoretical baggage and professional practice. The theoretical and methodological support of both professions came through the systematization of interventions carried out by pioneers in the field, the consolidation of evidence-based models and theoretical reflection.

This experience provides two results with great potential for the professional and academic ground in the psychosocial and socio-educational field. On the one hand, the evaluation of socio-educational intervention models with people in social exclusion in a multidisciplinary way. On the other, it systematizes and shares a consensual model for intervention from social services.

International and local literature in the field of social inclusion share these challenges, hence, this systematization provides technical criteria, theoretical bases, approaches and methodology that will surely shed light on professional dilemmas present in other territories.

The social and behavioral sciences provide interesting methods for evaluating practice and redefining procedures. The alliance built on this experience allows important steps to be taken for the social service sector. Experiences such as these can encourage the awakening of other methodological revision and systematization processes, contributing to the necessary debate and discussion on strategy assessment and adaptation of social services to new realities.

Keywords: social services, social exclusion, profiling, socio-educational, systematization.

\section{Introducción}

\section{Nuevos riesgos y perfiles para la intervención desde los servicios sociales}

Los servicios sociales se gestaron en España en un contexto muy distinto al actual. El pleno empleo masculino y la presencia de empleo estable en gran parte de los hogares aportaban cierta estabilidad económica en las familias. Del mismo modo, las familias tradicionales ofrecían cuidado y atención 
a menores y dependientes (Martínez Virto y Pérez Eransus, 2018). Desde hace unas décadas, la sociedad española se enfrenta a importantes cambios sociales vinculados a nuevos patrones de cuidado en las familias, así como a procesos de exclusión laborales afectados fuertemente por la precarización de las condiciones del mercado de trabajo, especialmente, tras la grave crisis del 2008. Por tanto, la capacidad integradora del empleo se ha visto fuertemente deteriorada. Igualmente, el aumento de la esperanza de vida y los cambios en las familias han incrementado la presión de los cuidados. Como resultado, la interrelación de estas necesidades ha incrementado, cuantitativa y cualitativamente, la demanda potencial de los servicios sociales en España.

En este contexto de nuevas necesidades sociales y mayor volumen de demanda, los servicios sociales de España se enfrentan a nuevos perfiles con procesos de exclusión de diversa gravedad e intensidad, especialmente en el campo de la incorporación social (Taylor-Gooby, 2004).

El impacto de la crisis del año 2008 supuso un fuerte incremento del desempleo y la precariedad laboral (Laparra y Pérez, 2012). La crisis, larga e intensa, generó procesos de exclusión largos y progresivos que no solo tuvieron consecuencias en la capacidad económica de las familias, sino que acarrearon fuertes consecuencias en el espacio de la salud y de las relaciones familiares (Martínez Virto, 2014). Más de una década después de la crisis económica, al menos uno de cada diez hogares en España ha vivido algún trastorno de salud metal o depresión. Según la encuesta longitudinal Foessa (2019), en el año 2018, uno de cada diez hogares manifestaba haber sufrido en los últimos diez años algún trastorno de salud mental o depresión (10.6 $\%$ ). Este porcentaje se incrementaba al 12.6 $\%$ en el caso de hogares con algún activo en desempleo y al $16.2 \%$ en caso de hogares con todos los activos en paro. Los datos muestran para 2018 que estas patologías inciden, con respecto a la población integrada, tres veces más entre los hogares en exclusión social y el doble en aquellos afectados por la precariedad.

En esta misma línea, el Informe sobre los Servicios Sociales de España (ISSE), realizado por el Consejo General del Trabajo Social en España y que para el año 2018 contó con una muestra total de 1490 testimonios de profesionales del Trabajo Social en España, cuantificó que más de 4 de cada 10 personas atendidas por los servicios presentan necesidades vinculadas a la exclusión social y la pobreza (Vicente et al., 2018). Este volumen se ha mantenido entre el 50-40\% del total del volumen de demanda entre los años 2013 y 2018, según muestran los informes anteriores (Lima et al., 2015).

Otra consecuencia de la crisis económica que afecta a los servicios, al margen de su demanda, fue la implementación de políticas y enfoques de austeridad en la intervención social (Pastor et al., 2019). Las políticas de austeridad afectaron la orientación de la intervención profesional, especialmente en aquellas áreas vinculadas a la protección de ingresos mínimos o la activación (Martínez Virto, 2019). En este sentido, se produce un incremento del discurso activador presente también en otros países como Reino Unido (Carter y Witworth, 2016) y un aumento de la participación de otros actores en la gestión de estos programas. El resultado ha sido un escenario con más demanda, un constreñimiento de la capacidad de protección de los servicios y, sobre todo, nuevos enfoques más punitivos contra la pobreza (European Social Network, 2015).

$\mathrm{Si}$ bien estos cambios han sido también frecuentes en otros países Europeos y cuentan con amplias evidencias en el ámbito internacional (Spolander et al., 2014), el impacto de la crisis en los servicios sociales ha sido más intenso en los países del Sur de Europa como Grecia, Italia o España (García y Rendueles, 2017; Garrett y Bertotti; 2016; Karagkounis, 2016; Pentaraki, 2017) En el caso de España, ello se debe, fundamentalmente, a tres 
factores que limitan la capacidad inclusiva de los entornos: el débil sistema de garantía de rentas, la fuerte precariedad laboral y una vivienda cara (Martínez Virto y Pérez Eransus, 2018). Estos factores contribuyen a la inestabilidad vital de las familias, ya que, en épocas de crisis y precariedad, la capacidad de resistencia familiar se ve muy afectada y las consecuencias psicosociales se relacionan con la falta de cobertura las necesidades básicas de supervivencia (rentas, empleo y vivienda) (Foessa, 2019). Todos estos factores concatenados han generado también efectos psicosociales como la pérdida de autoestima o síntomas de depresión, los cuales alejan a estas personas de los procesos de incorporación social y hacen más compleja y costosa la intervención en los servicios sociales (Martínez Virto, 2014).

Este aumento y la complejidad de la demanda potencial en los servicios sociales ha abierto una línea de debate que parecía extinta tras el proceso de universalización del sistema: la selección y priorización de perfiles para una intervención más adecuada, eficiente y eficaz. De este modo, la realidad de los servicios sociales en España se enfrenta, en este ámbito de la inclusión, a la necesidad de afrontar los problemas de organización y gestión para garantizar, en todos los territorios, respuestas eficaces a la población excluida (Martínez Virto, 2019). Ello queda ampliamente testado en el ISSE de 2018 que identifica los seis mayores retos a los que se enfrenta el sistema de servicios sociales en España para los próximos tres años y entre los que están: responder a las mayores demandas, mejorar la organización y adaptarse a las nuevas problemáticas (Vicente et al., 2018).

Algunos de estos retos nunca han sido resueltos y vienen originados desde la extensión de estas redes públicas de atención, pero otros han venido motivados e intensificados por estas primeras reacciones a las distintas crisis económicas. Esta situación ha motivado distintos procesos de revisión organizativa o normativa en regiones como Asturias, Comunidad Valenciana o Navarra (Aguilar, 2019;
Caravantes, 2020; CSDS, 2018). Con ello, se ha buscado responder de manera más eficiente y eficaz a este escenario, aplicando distintas estrategias organizativas como protocolos de atención adecuados, normativas más inclusivas o intensidades de intervención más ajustadas a la complejidad que requiere cada caso.

La identificación de criterios de urgencia y perfiles de personas usuarias ha demostrado en otros sistemas, como el sanitario, avanzar en protocolos de acción y trazar itinerarios de atención. En el ámbito de la intervención laboral es también una línea de trabajo avanzada, con ejemplo en países como Eslovaquia, Alemania o Irlanda (Blázquez, 2014; Mýtna, 2014; O'Connell et al., 2012; Rudolph y Konle-Seidl, 2005). En general, estos procesos se han destinado a medir la empleabilidad de las personas atendiendo a criterios como el sexo, nivel educativo o experiencia laboral, así como a otros factores como la condición de salud, motivación o redes sociales disponibles (Konle-Seidl, 2011). Igualmente, aunque para ello se han desarrollado métodos distintos, destaca el perfilado estadístico orientado a prevenir procesos de desempleo de larga duración en población de alto riesgo (Desiere, Langenbucher y Struyven, 2019).

De estas experiencias de perfilado de personas en desempleo o de los nuevos enfoques en el desarrollo de las organizaciones se extraen dos conclusiones que resultan de interés en el ámbito de los servicios sociales. La primera es que para superar las barreras de acceso al empleo no solo se debe superar la ausencia de competencias y experiencia laboral de las personas, sino trabajar otras necesidades sociales de los casos (Bazquez, 2014; Lopera y Echeverri, 2018). La segunda es que, más allá de las casuísticas de las personas, la eficiencia de las atenciones y sus resultados a medio y largo plazo se relacionan con los enfoques y estilos de intervención profesional (Duell y Kurekova (2013; O'Connell et al., 2012).

Este tipo de experiencias en el ámbito de servicios sociales no cuenta con extensa evidencia. Destaca una experiencia en la región 
de Castilla y León financiada por la Comisión Europea mediante el proyecto PACT (ProActive Case-based Targeted model). Mediante esta experiencia se avanzó en un método de diagnóstico social compartido entre servicios sociales y otras entidades de iniciativa social. Con esta información se trató de avanzar en la identificación de perfiles de usuarios/as atendidos (RIS, 2019). El objetivo, al igual que en la experiencia de Pamplona, era trabajar de manera proactiva desde los servicios sociales y prever la urgencia e intensidad de la atención (Martínez Virto y Pérez Eransus, 2018).

En definitiva, los cambios acontecidos requieren formas innovadoras de organización $\mathrm{y}$ atención desde los servicios sociales. Estas cuestiones se alinean también con los nuevos desafíos en la gestión del conocimiento en los centros de trabajo (Zalakain, 2016). La dificultad de acotar la acción, protocolizar el procedimiento, identificar las prioridades y marcar las intensidades de la intervención se debe, en buena parte, a la propia heterogeneidad de los casos y a las diferencias de oportunidades de inclusión en los territorios. Pero también, a la falta de cultura de evaluación y redefinición de los procesos.

A pesar del carácter territorial de estos desafíos, los gruesos de los nudos son compartidos por distintos territorios, no solo nacionales, sino también internacionales. La literatura internacional sobre la crisis y los servicios sociales alumbra necesidades similares en los distintos países (Spolander et al., 2014). Por ello, avances de consenso y sistematización que se emprendan en esta materia resultarán de gran interés para el aprendizaje entre territorios.

\section{La alianza entre la sistematización y la práctica profesional para la identificación de perfiles e intensidades}

Las profesiones de atención a las personas como la psicología o el trabajo social han nacido de la alianza teórica con la práctica profesional. La reflexión teórica, la sistemati- zación de la intervención realizada desde sus pioneras y la consolidación de los métodos de intervención con modelos basados en la evidencia han contribuido al soporte teórico y metodológico de estas profesiones (Berroeta, 2007; López Peláez y Segado, 2012).

La sistematización implica una reflexión profunda de la práctica profesional desde un análisis teórico, de enfoque, de adecuación a las necesidades y de organización profesional. Mediante este proceso se produce una evaluación de la actividad profesional impulsando, en algunos casos de inadecuación, la redefinición de los procesos de atención. En este sentido, la sistematización profesional juega una doble misión. Por un lado, evalúa la práctica profesional a partir del impacto de la acción o, lo que es lo mismo, la capacidad de respuesta e intervención de los métodos utilizados. Por otro lado, supone una metodología de difusión de resultados y métodos de intervención que contribuye a la trasferencia de conocimiento entre equipos, profesionales e incluso territorios (Cifuentes, 2010).

Los métodos de evaluación, basados en la evidencia y de la sistematización profesional, tienen un peso importante en la construcción teórica y práctica en las profesiones de atención a las personas. En el caso del trabajo social, aunque su trayectoria es joven comparada con otras disciplinas más arraigadas como la psicología, en los últimos años se ha incrementado la literatura académica que busca consolidar la aplicación y definición compartida de estos métodos de evaluación de la práctica profesional (Parrish, 2018). Sin embargo, son paradigmas que todavía encuentran barreras para ser implementados como un paso más de la intervención real entre los profesionales. De hecho, en otros países como Noruega, con amplia trayectoria en el diseño de modelos de acción avanzados, existen evidencias de cierta confusión en la utilización del concepto y su método (Ekeland et al., 2018).

A pesar de ello, crece la producción académica y la implicación profesional en este 
campo de la evaluación aplicada, animada por la consolidación de esta metodología en otras ciencias como la psicología. En los últimos años se han impulsado iniciativas internacionales que, bien desde la práctica como desde la investigación, han comenzado a sistematizar y difundir experiencias de intervención social. Destaca en este campo la Red de Practicas Sistematizadas (Reprasis) desde Latinoamérica o los informes de la Unión Europea que identifican prácticas innovadoras en materia de inclusión (Reuter, 2012).

La metodología cualitativa aplicada y desarrollada desde las ciencias psicosociales aporta importantes claves para la construcción de material empírico aplicado a la mejora de los procesos de gestión e intervención en los servicios sociales (Rubin y Babbie, 2009). En concreto, los métodos basados en la evidencia práctica o la observación sistemática pueden ser aliados de la investigación y evaluación de la práctica en profesional (Gordo y Serrano, 2008). Del mismo modo, Fortune et al. (2013) señalan cómo la evaluación de programas o dispositivos de intervención suponen un nuevo método de revisión de la práctica profesional que aporte nuevos conocimientos y claves para las decisiones organizativas desde las instituciones responsables. Es por ello que las potencialidades de estos métodos y herramientas generan la oportunidad de desarrollar un proceso de sistematización desde la reflexión y la investigación aplicada con importantes transferencias (Caparrós et al., 2017).

\section{Proceso metodológico de}

\section{sistematización y definición}

\section{de perfiles en los servicios sociales}

\section{de Pamplona}

Pamplona es una ciudad de algo más de 200000 habitantes, situada al norte de España, y es la capital de la Comunidad de Navarra.
El sistema de servicios sociales en España es una competencia autonómica que se desarrolla en colaboración con las entidades locales. La Comunidad Foral de Navarra es uno de los territorios pioneros en desarrollar y legislar el sistema de servicios sociales. Tras una primera normativa de implementación en el año 1983, inició un profundo proceso de revisión organizativa que dio lugar a una nueva ley (LF 15/2006) en el año 2006. Esta norma incorporaba criterios de atención diferenciados en el nivel de atención primaria en torno a cuatro programas diferenciados: (1) acogida y orientación, (2) incorporación social, (3) infancia y familia y (4) autonomía y dependencia. La experiencia que aquí se presenta se enmarca en el segundo de estos programas, el de incorporación social, y estuvo motivada por el fuerte incremento y diversificación de la demanda tras la crisis del año 2008.

Generalmente, la motivación a iniciar procesos de evaluación y readecuación de la práctica y la organización de los servicios se ve impulsada por fuertes cambios en las condiciones de trabajo que generan confusión e inseguridad a las profesionales. En el caso de Pamplona, al fuerte impacto de la crisis económica, se le sumó una insuficiente definición del modelo organizativo marcado en la LF 15/2006. Esta realidad resultó crítica tanto en la respuesta coherente y homogénea a la ciudadanía, como en la orientación de las profesionales a la gestión de las necesidades de la demanda. Como resultado, se inició un movimiento técnico que animó a comenzar un proceso de trabajo orientado a definir perfiles de atención y contenidos homogéneos que permitieran impulsar acciones adaptadas, derivaciones coherentes y una respuesta de calidad a la ciudadanía.

Cabe señalar que esta dificultad no solo se hizo palpable en la ciudad de Pamplona, sino que el cambio de escenario motivó un proceso de reflexión regional liderado por el Gobierno Autonómico (Aguilar, 2019). No obstante, fueron las profesionales del Programa de Incorporación de la ciudad de Pamplona las 
que iniciaron un proceso profundo y pionero de reflexión con importantes aportes a los distintos procesos de revisión de la atención que se estaban desarrollando tanto en Navarra como en otras regiones de España.

La selección del método de sistematización basado en un proceso de construcción de conocimiento colectivo y consensuado fue motivada por tres cuestiones fundamentales ya avanzadas en el inicio del texto. En primer lugar, la necesidad de identificar la población que llega al programa (características, necesidades e intensidad del deterioro); en segundo lugar, por la construcción de métodos de intervención consensuados y homogéneos entre la totalidad de profesionales del programa; y por último por el deseo de revisar y evaluar la eficiencia y adecuación de los modelos de intervención utilizados.

El proceso se inició en enero de 2018 y se prolongó durante 12 meses. Su desarrollo contó con dos fases diferenciadas. En primer lugar, se identificaron los distintos perfiles atendidos por el programa, sus necesidades principales y la intensidad de su deterioro. Este proceso ayudó a revisar la acción desarrollada por el programa teniendo en cuenta las siguientes categorías: población diana, tiempo de respuesta, tiempo de cita, intensidad de seguimiento, duración de la intervención, perfil profesional implicado y enfoque de intervención. Para ello, la muestra total de casos atendidos durante todo el proceso fue de 1077 casos (SIUSS, 2018). En cada encuentro quincenal se debatían los casos que aportaban cada una de las profesionales participantes en el grupo motor. La selección de los casos de alta estaba motivada por criterios como la edad, el sexo, la situación familiar, capital relacional, relación con el empleo y situación emocional. En aquellos casos de seguimiento se debatían dificultades de los procesos de intervención. Para poder contrastarlos, cada profesional aportaba fichas de registro con esta información a la sesión de grupo. En ella se discutían y contrastaban con la población atendida por el resto de profesionales del programa.
Posteriormente, y una vez definidos los perfiles y las tipologías de necesidad, se inició la segunda etapa del proceso en la que se acordaron la intensidad de la atención y el seguimiento a cada uno de los perfiles. Esta fase estuvo acompañada de un profundo proceso de reflexión y evaluación del método $\mathrm{y}$ enfoque de intervención del programa. Desde la primera fase se constató la gran variabilidad de métodos de intervención, no solo en relación con el tiempo, el perfil o la intensidad, incluso también con el enfoque. Por ello, en la fase dos se acordaron criterios e intensidades de atención más adaptados y homogéneos. Para ello, se definieron itinerarios de intervención protocolizados.

En este proceso de sistematización y definición psicosocial de perfiles e intensidades participaron un total de 19 profesionales, el total de las vinculadas al programa, entre las que se encuentran profesionales del trabajo social, la educación social o la intervención psicológica. Dentro de este grupo se creó una comisión de trabajo motor que lideró el proceso, formada por nueve profesionales, cuatro trabajadoras sociales, cuatro técnicas de educación social y una psicóloga, la única vinculada al programa en toda la ciudad.

En cada uno de estos encuentros se discutían los casos, las estrategias de intervención y la intensidad de seguimiento. De esta manera, comenzó el proceso de revisión de la práctica profesional que se estaba desarrollando en el programa, iniciando un debate de adecuación y evaluación de estos contenidos a partir de las siguientes categorías: nuevos perfiles, características psicosociales de priorización o urgencia, criterios de intensidad y circuitos de atención con las personas usuarias.

Una vez construidos los primeros avances desde el grupo motor de nueve profesionales, los acuerdos y procedimientos fueron enviados al resto de profesionales del programa. El objetivo era someter a contraste y nuevo debate los procedimientos propuestos. Una vez contrastado y consensuado, el resultado fue sistematizado para 
generar los procedimientos de intervención en el programa.

La toma de decisiones en este campo es controvertida y no está exenta de costes. La heterogeneidad que caracteriza a las situaciones de exclusión social ha sido un obstáculo tradicional para el establecimiento de perfiles de atención psicosocial en el campo de los servicios sociales. Sin embargo, las profesionales valoran con mucha satisfacción la toma de decisiones y los consensos establecidos en el proceso, ya que han contribuido a sentar las bases de su intervención.

Los resultados suponen un paso de gigante en la definición de los procesos de incorporación social. Si bien son propuestas que se ubican en el modelo organizativo de la atención primaria de los servicios sociales en Pamplona y Navarra, los consensos alcanzados también serán de utilidad para otros territorios que se enfrentan a tendencias exclusógenas y dificultades de atención similares.

\section{Perfiles psicosociales e intensidad para la atención a personas en exclusión social en los servicios sociales de Pamplona}

La heterogeneidad de perfiles y atención en los servicios sociales es una dificultad que excede la realidad de Pamplona. En estos años de fuerte demanda en los servicios sociales por la crisis económica, el programa de incorporación de servicios sociales se convirtió en "un cajón de sastre" al cual se derivaban, fundamentalmente, aquellos casos que, independientemente de su situación psicosocial, requerían oportunidades de inclusión. Este contexto aviva dos de los fuertes debates que existen en torno a los servicios sociales: su población diana y la definición de itinerarios de intervención. Por ello, este proceso ha ofrecido la oportunidad de evaluar, redefinir y sistematizar la práctica profesional. Como resultados principales destacan, por un lado, la definición de la población a atender y, por otro, la identificación de intensidades y enfo- ques de intervención para cada uno de estos perfiles. Se detallan a continuación los resultados de este proceso de sistematización y perfilado.

\section{Población diana, orientación diagnóstica y fases de los itinerarios de incorporación sociolaboral}

Desde el inicio del proceso se identificó la necesidad de revisar ampliamente tres aspectos que contribuían a la heterogeneidad de las respuestas profesionales: su población diana, la orientación diagnóstica y las fases de los itinerarios. A partir de estas barreras se acordaron las primeras bases que permitirían ir definiendo los perfiles psicosociales y las intensidades de los casos.

En torno a su población diana, el programa de incorporación social revisó los casos tratados entre los años 2017 y 2018 identificando como beneficiarias potenciales a personas o unidades familiares que se encuentran en una situación de fragilidad o vulnerabilidad de exclusión social. Para ello, se tomó en consideración tanto su situación en distintos aspectos como el empleo, la vivienda, el sociosanitario, la convivencia, la educación, las redes sociales o las necesidades básicas, así como, su ámbito personal y psicosocial.

Por otro lado, las herramientas diagnósticas utilizadas en la elaboración del diagnóstico psicosocial tenían ampliamente desarrollados campos de acción como la vivienda, el empleo, la salud y los consumos, el cuidado o la situación familiar. Sin embargo, no incluían otros como las competencias personales de las usuarias, tanto en habilidades sociales o comprensión como en educación y capacitación. La ausencia de herramientas básicas personales de manejo en la vida cotidiana era identificada por las profesionales como una de las principales barreras de incorporación de las personas, requiriendo un fino diagnóstico que permitiera abordarlas.

Como resultado, se reformularon las bases diagnósticas y su enfoque metodológico. Para 
el diagnóstico del ámbito personal o psicosocial se acordó atender dos áreas fundamentales. En primer lugar, las competencias y habilidades sociales de las personas, entendidas como los comportamientos instrumentales que la persona tiene para resolver situaciones cotidianas, o en su carencia, las dificultades o conflictos presentes en su vida. Este punto estaba ligado a la facilidad de comprensión y comunicación, así como, al autocontrol de las emociones. Entre ellas destacaban la ausencia de competencias psicosociales básicas de interacción y comunicación, las competencias cognitivas de lenguaje o comprensión, las instrumentales que permiten la interacción con el medio, relaciones sociales o acceso a otros recursos de ocio o salud, así como otras habilidades personales de autocuidado, convivencia con otras personas o la capacidad de tomar decisiones de manera autónoma. En este campo también se identificó la capacitación de la persona en relación a su nivel educativo o formación. El trabajo en este ámbito iría orientado a trabajar acciones encaminadas a la adquisición de habilidades previas y hábitos prelaborales a través de la metodología del acompañamiento social.

En relación con el enfoque del programa, se constató un fuerte acento en la metodología de trabajo individual, viendo necesario repensar las acciones tanto a nivel individual y familiar como de carácter grupal o comunitario. En el marco de este proceso se llegó al acuerdo de que la intervención a nivel individual y familiar tendría tres objetivos importantes: (1) promover la adquisición y desarrollo de competencias que desarrollen una creciente autonomía personal y social; (2) estimular la responsabilidad sobre su propio proceso de desarrollo personal y promoción social como miembro de una comunidad; y (3) facilitar el acceso a idénticas oportunidades que el resto de la población. En el caso de la intervención a nivel grupal o comunitario, se plantearon como horizonte el objetivo de crear, potenciar o mantener la red de apoyos familiares y vecinales en el entorno social más próximo, promover acciones educativas $\mathrm{y} / \mathrm{o}$ formativas que favorezcan la incorporación social y sensibilizar a la ciudadanía sobre las situaciones de exclusión social para fomentar una sociedad más igualitaria y solidaria.

Por último, una vez identificadas estas limitaciones en la delimitación y los objetivos del programa, la redefinición de la intervención se orientó a homogeneizar la duración de la atención, el contenido y las fases de los procesos de intervención con las familias e individuos atendidos.

En relación con la duración de la intervención se acordó un tiempo máximo de dos años. Comenzaría con una primera entrevista de encuadre de la intervención de forma entendible y con un lenguaje adaptado, para explicar en qué consiste el Programa de Incorporación Social, su equipo multidisciplinar de trabajo (trabajo social, integración social y psicología) y con qué recursos cuenta (acompañamiento, formación sociopersonal y psicológica, etcétera). En este primer encuentro se aclararía tanto la demanda y las expectativas como el grado de motivación de la persona para iniciar el proceso de incorporación.

En el siguiente encuentro se diseña el plan de intervención. Para ello, tanto el equipo técnico como la persona usuaria acuerdan el diagnóstico y los pasos a seguir en el proceso. En él se incluyen objetivos, actuaciones, recursos necesarios, pronóstico, calendarización y la frecuencia de atención. En general, los tiempos de revisión del caso serán, al menos, cada seis meses y tras cuatro revisiones en profundidad (dos años de intervención). Igualmente se realizará una valoración sobre la continuidad del caso o su finalización. En esa fase final se evaluará la situación con respecto a la partida, los objetivos cumplidos y los pendientes en los distintos ámbitos, las circunstancias de mejora, estancamiento o empeoramiento, los aspectos positivos y las dificultades encontradas, y los recursos utilizados. El seguimiento y la evaluación se recogen en el plan de caso, junto a los compromisos de ambas partes, y todo ello forma parte del acuerdo de incorporación. 
La definición de estas bases del programa en la primera fase del proceso de revisión implicó un impulso de la acción hacia la intervención integral que, con mayor o menor nivel de intensidad, y mediante el acompañamiento psicosocial y socioeducativo, pretende alcanzar un mayor nivel de bienestar de la persona en sus distintos ámbitos de vulnerabilidad. Este acompañamiento persigue el objetivo de construir una relación de ayuda con las personas atendidas que pone en el centro de la acción a la propia persona, protagonista de los tiempos de itinerario y la toma de decisiones. Tras este primer ejercicio de definición de objeto y métodos, la segunda fase avanzó en la identificación de perfiles psicosociales e intensidades de la atención para cada situación de dificultad.

\section{Perfiles psicosociales e intensidades de atención}

Como se ha señalado anteriormente, la población diana a la que se dirige el programa está compuesta por personas de entre 16 y 65 años que necesitan un apoyo en el proceso de incorporación social. No obstante, la intensidad de la atención se diferencia en torno a aquellas personas que permanecen donde solo se precisa la voluntariedad y aquellas que van a participar en un acompañamiento socioeducativo que requiere más motivación, disposición para el cambio y un manejo básico del idioma. Por otro lado, también se ofrece apoyo psicológico con distinta intensidad a casos que lo requieran para emprender su proceso de inclusión.

A pesar de esta diversidad de situaciones de necesidad, el programa nunca había realizado una sistematización de perfiles psicosociales. Ello suponía una fuerte barrera para la definición de procesos de trabajo diferenciados a partir de las necesidades de las personas. Por eso, uno de los resultados más significativos de este proceso ha sido la previa identificación y posterior definición de perfiles de necesidad. Para ello, se concretan tres enfoques de atención vinculados a las necesidades de cada caso: atención básica, acompañamiento socioeducativo e intervención psicológica (ver Tabla 1).

Dentro de las usuarias potenciales del programa de incorporación con atención básica se identifican siete grupos. En primer lugar, las personas que provienen de un recurso residencial y han tenido una intervención intensa o proceso de acompañamiento. Algunos ejemplos son las personas sin hogar, de comunidades terapéuticas, en recursos residenciales de Tercer Sector, personas que han salido de prisión o de pisos de acogida para personas refugiadas, entre otras. En segundo lugar, se identifica a las familias que han padecido violencia de género y salen de recursos especializados de protección. Por otro lado, los jóvenes de programa de protección de menores actualmente sin apoyos o con apoyos inadecuados. En cuarto lugar, las personas con problemas de salud mental, discapacidades y/o consumos sin apoyos familiares ni institucionales. El quinto grupo son colectivos migrantes con necesidad de intervención intensiva y abordaje grupal y/o comunitario (mujeres nigerianas, población romaní, etcétera). En sexto lugar, las personas o familias usuarias que sufren un momento concreto de crisis o vulnerabilidad y requieran una atención integral y global. Por último, se identifica a aquellas personas en desempleo de larga duración, o sin ingresos, que necesiten apoyo sociolaboral.

Entre los casos que requieren acompañamiento socioeducativo más intenso estarían las personas o familias que se enfrentan a un proceso de exclusión mayor. Destacan las personas que han pasado por un recurso de acogida que precisan un acompañamiento socioeducativo. Por otro lado, las personas que estando en algún recurso de formación, de alojamiento (personas sin hogar o en vivienda de emergencia social), de salud (deshabituación de sustancias o tratamiento) u otros que requieran un apoyo adicional para su proceso de incorporación. En tercer lugar, personas en 
proceso de empoderamiento con conciencia y/o deseo de cambio en su ámbito personal que necesiten apoyo en el proceso, usuarias en proceso adaptativo a nueva situación o personas en exclusión social grave con deseo de cambio. También personas con enfermedad mental con conciencia de su enfermedad que requieren apoyo concreto para evitar el empeoramiento del caso. Por último, personas que se encuentren en procesos educativos con objetivos concretos y vinculados a la gestión de emociones, habilidades sociales, etcétera.

Tabla 1

Clasificación de los perfiles psicosociales de atención, por programa de intervención.

\begin{tabular}{|c|c|}
\hline $\begin{array}{r}\text { Ár } \\
\text { inter }\end{array}$ & Perfiles de atención \\
\hline $\begin{array}{l}\text { Programa de } \\
\text { Incorporación. } \\
\text { Atención básica }\end{array}$ & $\begin{array}{c}\text { Personas que provienen de recurso residencial y requieran apoyo (sin } \\
\text { hogar, comunidades terapéuticas, residencias temporales, prisión o pisos de } \\
\text { acogida). } \\
\text { Personas que provienen de recursos especializados de atención en casos de } \\
\text { violencia de género. } \\
\text { Personas que provienen de recursos de protección de menores sin apoyos o } \\
\text { con apoyos inadecuados. } \\
\text { Personas afectadas por salud mental, discapacidades y/o consumos, sin } \\
\text { apoyos familiares ni institucionales y con falta de habilidades y de recursos. } \\
\text { Personas que perciben prestaciones de garantía de renta donde requieren } \\
\text { hacer una acción de activación durante ese tiempo (laboral, formativa o } \\
\text { personal). } \\
\text { Colectivos específicos asentados en el territorio con necesidad de interven- } \\
\text { ción intensiva y abordaje grupal y/o comunitario (mujeres nigerianas, pobla- } \\
\text { ción romaní, etcétera.) } \\
\text { Personas o familias usuarias de la unidad de barrio que en un momento } \\
\text { concreto de crisis o vulnerabilidad requieran una atención integral y global. }\end{array}$ \\
\hline $\begin{array}{l}\text { Acompaña- } \\
\text { miento socioe- } \\
\text { ducativo }\end{array}$ & 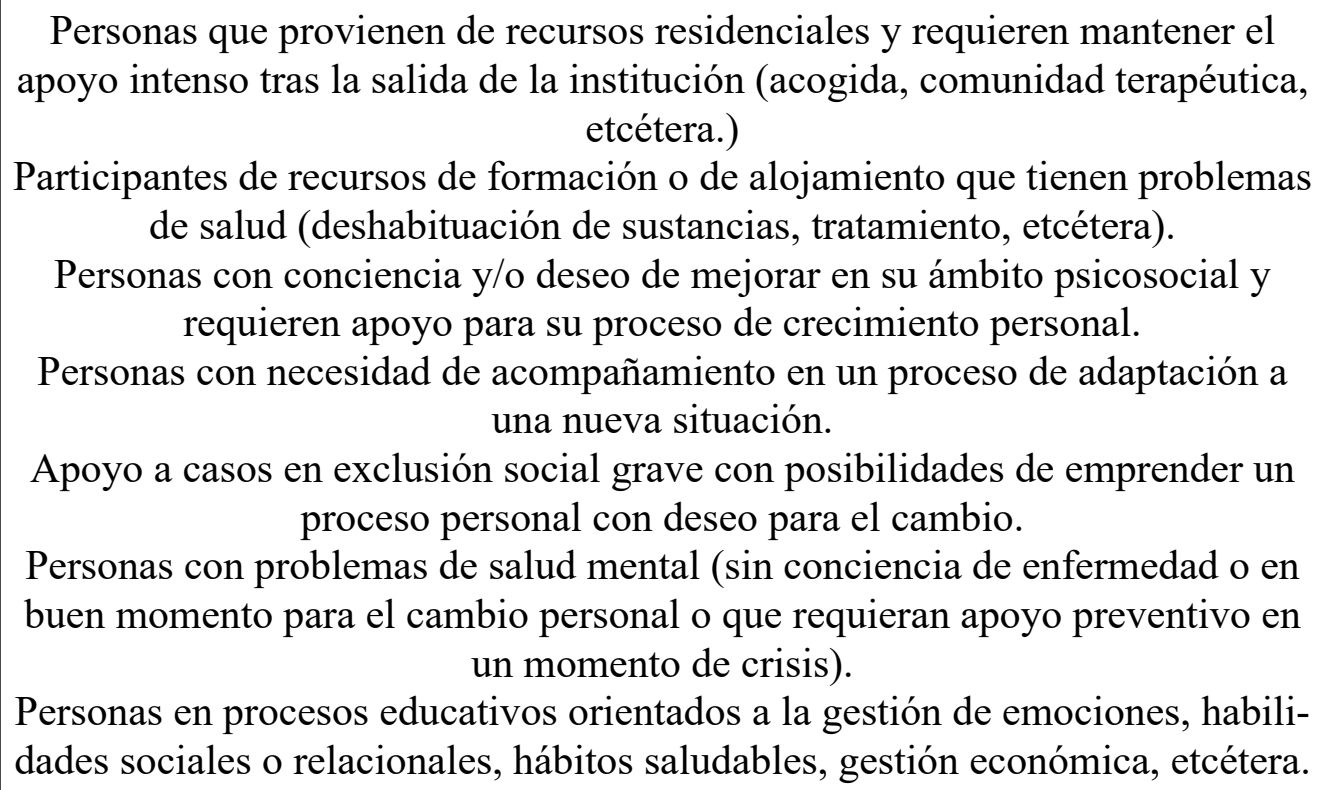 \\
\hline
\end{tabular}




\begin{tabular}{c|c}
$\begin{array}{c}\text { Área de } \\
\text { intervención }\end{array}$ & Perfiles de atención \\
\hline $\begin{array}{c}\text { Intervención } \\
\text { psicológica }\end{array}$ & $\begin{array}{c}\text { Intervención breve (10 sesiones, 6 meses): Elaboración de duelos, pérdidas, } \\
\text { Dificultades de adaptación a cambios vitales importantes (emancipación, } \\
\text { migración, separación de pareja, etc.), Crisis de ansiedad o angustia. } \\
\text { Intervención de intensa (30 sesiones, año y medio): Sufrimiento personal, } \\
\text { Conductas disfuncionales, Pensamientos y relaciones no adaptativas en } \\
\text { posible relación con: Desestructuración familiar, Institucionalización en } \\
\text { infancia o adolescencia, medidas de protección (pisos acogimiento familiar, } \\
\text { etc.), Violencia de género, sospecha de abusos, Toxicomanías, formas de vida } \\
\text { marginal, etcétera. }\end{array}$ \\
\hline
\end{tabular}

Fuente: Elaboración propia a partir de los criterios definidos por las profesionales en el proceso de sistematización.

El programa ofrece también una unidad de apoyo psicológico para los casos más intensos o que están en la fase socioeducativa. Desde este enfoque se establecen dos tipos de apoyo. Por un lado, el apoyo breve que durará entre 10 sesiones y seis meses orientado a personas que están viviendo un proceso de duelo, ansiedad o dificultades de adaptación a cambios vitales (emancipación, migración o separación de pareja). Por otro lado, un apoyo intenso con 30 sesiones a desarrollar en 18 meses para las personas o familias que viven momentos de sufrimiento personal, conductas disfuncionales o pensamientos y relaciones no adaptativas como consumos o enfermedades con conciencia, desestructuración familiar, medidas de protección a la infancia o adolescencia, violencia de género, sospecha de abusos o formas de vida marginal.

Si bien esta primera clasificación de perfiles, a partir de la necesidad que presenta el caso, ha supuesto un gran avance en la concreción de la acción profesional, el trabajo con personas en dificultad requiere desarrollar evaluaciones frecuentes para adecuar o flexibilizar la atención. Para ello se identifican tres grados de intensidad en la atención: alta, media y baja (ver Tabla 2).

Los casos de mayor intensidad son aquellos que requieren mucho acompañamiento por encontrarse en una crisis como un proceso de duelo, abandono o pérdida (vivienda, familiares, rupturas traumáticas), las situaciones de maltrato en procesos vitales (infancia, género, tercera edad, discapacidad), casos con diagnóstico de enfermedades graves propias o de familiares cercanos, las situaciones de pérdida de empleo en edades avanzadas o aquellas personas que han abandonado un recurso de institucionalización, etcétera. Estos casos serán preferentes y con nivel de atención flexible. En principio se identifica una atención mínima quincenal y un contacto frecuente (teléfono, mail. etcétera). La motivación del caso marcará la intervención interdisciplinar en el Equipo de Acompañamiento, formado por una Trabajadora Social y una Técnica de Integración Social. Contará, por supuesto, con el apoyo de la figura de psicología, dadas las potencialidades que implica el trabajo alineado entre disciplinas (Bedoya-Gallego et al., 2019). 
Tabla 2

Grados de intensidad en la atención psicosocial.

\begin{tabular}{|c|c|c|}
\hline & Criterios de definición & Intensidad de la atención \\
\hline Alta Intensidad & $\begin{array}{l}\text { Momento de la crisis, } \\
\text { gran necesidad de acom- } \\
\text { pañamiento. }\end{array}$ & $\begin{array}{l}\text { Situación preferente y con intensidad } \\
\text { flexible. Atención mínima quincenal, y } \\
\text { contacto frecuente telemático. } \\
\text { Intervención interdisciplinar (TIS, TS, } \\
\text { Psicología) marcada por el estado de caso. }\end{array}$ \\
\hline Media Intensidad & $\begin{array}{l}\text { Necesidades de acompa- } \\
\text { namiento psicosocial y } \\
\text { socioeducativo. }\end{array}$ & $\begin{array}{l}\text { Atención mínima programada: TIS (quin- } \\
\text { cenal), TS (mensual), psicología (en } \\
\text { función del caso) }\end{array}$ \\
\hline Baja Intensidad & $\begin{array}{l}\text { Casos estables, que } \\
\text { requieren seguimiento. }\end{array}$ & $\begin{array}{l}\text { Atención programada mínima cada tres } \\
\text { meses. }\end{array}$ \\
\hline
\end{tabular}

Fuente: Elaboración propia a partir de los criterios definidos por las profesionales en el proceso de sistematización.

Los casos de intensidad media serán aquellos en proceso de intervención que ya no se encuentran en momento de crisis, sino en fase de trabajo para el alcance de los objetivos. Las acciones que requieran acompañamiento social serán apoyadas por la profesional del trabajo social y aquellas de carácter socioeducativos por las profesionales de integración social. Para estos casos se marca una frecuencia de atención mínima quincenal para las acciones socioeducativas y mensual para las necesidades sociales. El apoyo psicológico se valorará en función del caso.

Como casos de intensidad baja se identifican aquellas situaciones estables que ya no requieren acompañamiento, pero sí un seguimiento hasta que el caso adquiera una estabilidad y autonomía en los objetivos planteados. Para ello, se cuenta con una atención programada mínima cada tres meses. La ubicación de los casos, en uno u otro nivel de intensidad, son valorados por el equipo multidisciplinar bajo criterios: el momento vital en el que se encuentra y las necesidades de acompañamiento que requiere. En los casos con los que se está trabajando, la evolución y trayectoria de los itinerarios marcará su paso por uno u otro grado de intensidad. Para ello, el Equipo de Acompañamiento (EA) se reunirá, al menos, una vez cada 15 días para coordinación y valoración de los casos comunes y, con la figura de psicología, como mínimo cada tres meses.

En definitiva, el proceso de evaluación ha tenido como resultado una sistematización de la acción profesional que sienta las bases, contenidos y criterios para la atención profesional. Este resultado, trabajado desde el consenso y aportación colectiva, supone un importante avance en materia de intervención psicosocial. Si bien la definición de perfiles no ha estado exenta de dificultades, los resultados presentados son el sustento de una nueva forma de atención más adecuada a las necesidades psicosociales actuales y más homogénea entre profesionales. Sin duda, los resultados superan el impacto en el programa, ya que caminan hacia una respuesta más eficiente en la atención desde los servicios sociales. La investigación aplicada tiene resultados importantes en la consolidación del programa, pero el trabajo con las personas requiere de la frecuente actualización de los métodos de trabajo. Por tanto, no es un proceso que acaba, 
pero sí es una nueva forma de trabajo que anima a la comunidad profesional a iniciar una nueva cultura de evaluación y sistematización de la práctica profesional.

\section{Conclusiones}

La crisis económica ha supuesto un fuerte golpe para el sistema de servicios sociales en España. Ello ha implicado no solo la complejidad de las necesidades psicosociales y la carga de gestión en el trabajo desde los servicios sociales púbicos, sino que, además, diversas investigaciones apuntan a que ello ha implicado un cambio en la acción profesional y los criterios de intervención, reorientando el trabajo de acompañamiento socioeducativo a una respuesta más rápida y menos trasformadora. Estos aspectos han intensificado tradicionales tensiones en la profesión vinculadas con la definición de funciones, la población diana, los procesos de trabajo o la evaluación de la intervención social.

La territorialización de los servicios en España ha tenido importantes beneficios en la cercanía de las respuestas a las personas, pero también ha contribuido a crear una fuerte fragmentación de la red. A lo largo de estos años, en los servicios sociales de atención primaria se han desarrollado metodologías de trabajo adaptadas a los entornos y se han diseñado nuevos recursos que han tenido un carácter innovador y novedoso. El conocimiento profesional acumulado en los servicios sociales es uno de los pilares de la atención a las personas. Sin embargo, la fragmentación de la red y una escasa cultura de la sistematización profesional han impedido la trasferencia entre territorios.

Desde el programa de Incorporación Social de los servicios sociales del Ayuntamiento de Pamplona se identificaron diversos procesos que dificultaban la atención. Responder a estos desafíos requería una revisión compartida y consensuada que adecuara el contenido y la orientación de los procesos de atención a las necesidades reales de la ciudadanía.
Ante este reto, las profesionales del programa iniciaron un proceso de reflexión y definición de perfiles e intensidades en la atención que es sistematizado en este trabajo.

El proceso de evaluación y sistematización de la acción profesional desarrollado en Pamplona supone un gran avance en la conquista de los desafíos señalados. La delimitación del programa, los procedimientos de gestión o la definición de la población diana son claves para ordenar y definir la acción del programa, especialmente tras el embate de la crisis y la diversificación psicosocial de la demanda recibida.

Este proceso aporta tres resultados con grandes potencialidades para la trasferencia al sector profesional y académico. Por un lado, la evaluación de la acción profesional con posibles aportes en la revisión de la intervención socioeducativa con las personas en exclusión social; por otro, la sistematización de una acción revisada, consensuada y adaptada a las necesidades actuales que busca superar las limitaciones de modelos anteriores vinculados a los servicios sociales; y por último, constata las potencialidades que ofrecen los procesos de evaluación y consenso profesional para la propia planificación de las políticas públicas o los programas de intervención.

La literatura internacional y estatal en los campos de la inclusión social comparte malestares, retos y desafíos. Por ello, esta sistematización aporta criterios técnicos, bases teóricas, enfoques y metodología que seguro arrojan luz a dilemas profesionales presentes en otros territorios. En concreto, el perfilado psicosocial de usuarias, la definición de intensidades de atención educativa o los tiempos de seguimiento implican una hoja de ruta que puede ser sustrato de otros programas o acciones orientadas a la incorporación.

Como se ha visto, las ciencias sociales y del comportamiento aportan interesantes métodos para la evaluación de la práctica y la redefinición de procesos. La alianza construida en esta experiencia permite dar pasos importantes para el sector de servicios sociales. Si bien 
los resultados y el propio proceso responden a una realidad territorial concreta, los criterios acordados aportan un sustrato muy importante para la lucha contra la exclusión social. Por ello, además de los aportes metodológicos al propio sector, aporta la trasferencia de resultados, contribuye a la planificación o diseño más ajustado de las políticas sociales y animan a la innovación profesional.

La experiencia profesional acumulada en el sector es importante y la implicación profesional ha sido siempre bandera de sus acciones. Por ello, experiencias como estas pueden animar a despertar otros procesos de revisión y sistematización metodológica, contribuyendo al necesario debate y discusión sobre las estrategias de evaluación y adecuación de los servicios sociales a las nuevas realidades y necesidades psicosociales.

\section{Referencias bibliográficas}

Aguilar, M. (2019). Apuntes para la reordenación de la atención primaria de servicios sociales de Navarra. Pamplona: Gobierno de Navarra.

Bedoya-Gallego, D.; Buitrago-Duque, C. y Vanegas-Arbeláez A. (2019). Transdisciplinariedad en salud mental: Una propuesta de modelo de formación. Interdisciplinaria, 36(1), 119-132. https://doi.org/10.16888/interd.36.1.9

Berroeta, H. (2007). Recursos para la planificación y sistematización de intervenciones psicosociales y comunitarias. En J. Alfaro y H. Berroeta, Trayectoria de la psicología comunitaria en Chile (pp. 345-372). Valparaíso: Universidad de Valparaíso.

Blázquez, M. (2014). Skills-based profiling and matching in PES Analytical Paper. PES-to-PES Dialogue. The European Commission Mutual Learning Programme for Public Employment Services. Brussels: DG Employment, Social Affairs and Inclusion.

Caparrós, N., Carbonero, D. y Raya, E. (2017). Construir conocimiento desde la práctica. Ejemplos de sistematización en Trabajo Social. Interacción y perspectiva: Revista de Trabajo Social, 7(1), 61-79.
Caravantes López de Lerma, G. (2020). El derecho de participación en las políticas sociales: el proceso participativo para la elaboración de la Ley valenciana de Servicios Sociales. Cuadernos De Trabajo Social, 33(1), 99-113.

Carter, E. y Whitworth, A. (2017) Work Activation Regimes and Well-being of Unemployed People: Rhetoric, Risk and Reality of Quasi-Marketization in the UK Work Programme. Social Policy \& Administration, 51, 796- 816. https://doi.org/10.1111/ spol.12206.

Cifuentes, R. (2010). Mediaciones en la implementación de políticas sociales sectoriales. Lecciones aprendidas desde la sistematización de cuatro experiencias. Revista Tendencias \& Retos, 15, 121-148.

Consejería de Servicios y Derechos Sociales (CSDS) (2019). Documento de bases para la elaboración de una nueva ley de servicios sociales del Principado de Asturias. Principado de Asturias: Consejería de Servicios y Derechos Sociales. https://www. socialasturias.es/servicios-sociales/normativa/documento-de-bases-nueva-ley-de-ser-

vicios-sociales-del-principado-de-asturias_1602_737_2503_0_1_in.html

Desiere S., Langenbucher, K. y Struyven, L. (2019). Statistical profiling in public employment services.An international comparison. Social, Employment and Migration Working Papers, 224, OECD.

Duell, N. y Kureková, L. (2013). Activating Benefit in Material Need Recipients in the Slovak Republic. CELSI Research Report, 3. Bratislava.

Ekeland, T. J., Bergem, R. y Myklebust, V. (2018). Evidence-based practice in social work: perceptions and attitudes among Norwegian social workers. European Journal of Social Work, 22(4), 611-622. https://doi.org/10.1080 /13691457.2018.1441139

European Social Network ESN (2015). Public social services in crisis: challenges and responses. From 2008-2014: a response from ESN members. Report for European Social Network. https://www.esn-eu.org/sites/default/ 
files/publications/2015_Public_Social_ Services_in_Crisis_report_-_FINAL.pdf

Foessa (2019). VIII Informe sobre Exclusión y Desarrollo Social de España. Madrid: Fundación Foessa.

Fortune, A. E., Reid, W. J. y Miller, R. L. (2013). Qualitative Research in Social Work. Maryland: Columbia University Press.

García, S. y Rendueles, C. (2017). Hacia un nuevo Trabajo Social crítico: presentación del monográfico: el gobierno de los social en la era neoliberal. Cuadernos de Trabajo Social, 30, 243-260.

Garrett, P. M. y Bertotti, T. F. (2016). Social work and the politics of 'austerity': Ireland and Italy. European Journal of Social Work, 20(1), 29-41.

Gordo, A. J. y Serrano, A. (2008). Estrategias y prácticas cualitativas de investigación social. Madrid: Pearson Educación.

Karagkounis, V. (2016). Social work in Greece in the time of austerity: challenges and prospects. European Journal of Social Work, 20(5), 651-665. https://doi.org/10.1080/13691457.20 16.1255593

Konle-Seidl, R. (2011). Profiling systems for effective labour market integration. Use of profiling for resource allocation, action planning and matching. Bruselas: DG Employment, Social Affairs and Inclusion.

Laparra, M. y Pérez-Eransus, B. (2012). Crisis y fractura social en Europa: Causas y efectos en España. Colección de estudios sociales, 35. Barcelona: Obra social La Caixa.

Lima Fernández, A. I. (2015) (coord.). II Informe sobre los Servicios Sociales en España (ISSE II). Madrid: Consejo General de Trabajo Social de España.

Lopera Arbeláez, I. y Echeverri Álvarez, J. (2018). Libertad y desarrollo humano en las organizaciones. Interdisciplinaria, 35(2), 395-408. https://doi.org/10.16888/interd.2018.35.2.9

López-Pélaez, A. y Segado, S. (2012). Teoría, método y práctica: perspectivas sobre la investigación en el ámbito de la intervención social. Valencia: Nau Libres.

Navarra (2006). Ley Foral 15/2006, del 14 de diciembre, de Servicios Sociales. Pamplona:
Parlamento de Navarra. http://www.lexnavarra.navarra.es/detalle.asp? $\mathrm{r}=10855$

Martínez Virto, L. (2014). Sobreviviendo a la crisis: estrategias de los hogares en dificultad. Barcelona: Ediciones Bellaterra.

Martínez Virto, L. y Pérez Eransus, B. (2018). The primary care model of Social Services to debate: dilemmas and professional reflections around the case of Navarre. Cuadernos de Trabajo Social, 31(2), 333-343.

Martínez Virto, L. (2019). Nueva pobreza, precariedad y rentas mínimas: respuestas para incentivar el empleo en el actual contexto sociolaboral. Cuadernos de Relaciones Laborales, 37(1), 155-175.

Mýtna Kureková, L. (2014). Review of Profiling Systems, Categorization of Jobseekers and Calculation of Unit Service Costs in Employment Services. Implications and Applications for Slovakia. Bratislava: EPIC. NGO for employment services.

O’Connell, P. J., McGuinness, S. y Kelly, E. (2012). The Transition from Short to Long Term Unemployment: A Statistical Profiling Model for Ireland. The Economic and Social Review, 43(1), 135-164.

Taylor-Gooby, P. (2004). New Risks, New Welfare: The Transformation of the European Welfare. Oxford: Oxford University Press.

Parrish, D. E. (2018). Evidence-Based Practice: A Common Definition Matters. Journal of Social Work Education, 54(3), 407-411.

Pastor Seller, E., Verde Diego, C. y Lima Fernández, A. I. (2019). Impact of neo-liberalism in Spain: research from social work in relation to the public system of social services, European Journal of Social Work, 22(2), 277-288. https://doi.org/10.1080/13691457.20 18.1529663

Pentaraki, M. (2017). Practicing social work in a context of austerity: experiences of public sector social workers in Greece. European Journal of Social Work, 22(3), 376-387.

Red Inclusión Social RIS (2019). Herramientas de diagnóstico de situaciones de vulnerabilidad y/o exclusión social. Madrid: Ministerio de Sanidad, Consumo y Bienestar Social 
Reuter, C. (2012). Facing New Challenges: Promoting active inclusion through social innovation. SOLIDAR, European Network of NGOs, 56. Bruselas: Unión Europea. https://www.solidar.org/system/downloads/ attachments/000/000/045/original/56_solidarbriefing_social_innovation_active_inclusion-2.pdf?1457601194

Rubin, A. y Babbie, E. (2009). Research Methods for Social Work. Belmont: Brooks/Cole.

Rudolph, H. y Konle-Seidl, R. (2005). Profiling for Better Services. Report on the European Profiling Seminar. Nuremberg: Institute for EmploymentResearch.http://213.241.152.197/ veranstaltungen/2005/Profiling_Report_2005_ English.pdf

SIUSS (2018). Sistema de Información de Usuarios de Servicios Sociales. Pamplona: Ayunta- miento de Pamplona.

Spolander, G., Engelbrecht, L., Martin, L., Strydom, M., Pervova, I., Marjanen, P., Tani, P., Sicora, A. y Adaikalam, A. (2014). The implications of neoliberalism for social work: Reflections from a six-country international research collaboration. International Social Work, 57(4), $301-312$.

Vicente, E., Arreondo, R. y Rodríguez, C. (2018). III Informe sobre los Servicios Sociales en España. Consejo General del Trabajo Social. https://www.cgtrabajosocial.es/files/ 5de783c0056f8/ISSE_III_WEB.pdf

Zalakain, J. (2016). Tendencias en la gestión del conocimiento en los servicios sociales. https:// blog.siis.net/2016/11/tendencias-gestion-conocimiento-servicios-sociales/.

Recibido: 24 de octubre de 2019 Aceptado: 23 de febrero de 2021 